\title{
Berufe und soziale Ungleichheit - Thematische Einführung und Inhalte des Sonderhefts
}

\author{
Christian Ebner · Andreas Haupt • Britta Matthes
}

Online publiziert: 14. Juli 2020

(C) Der/die Autor(en) 2020

Zusammenfassung Die Untersuchung der Bedeutung des Berufs für die soziale Ungleichheit hat eine lange Tradition. In diesem einleitenden Beitrag werden die im hier vorliegenden Sonderheft der Kölner Zeitschrift für Soziologie und Sozialpsychologie gesammelten Aufsätze in den Kontext der bisherigen Berufsforschung gestellt. Der Anspruch des Sonderhefts besteht darin, Berufe nicht wie andernorts als „Blackbox“ zu betrachten, sondern explizit danach zu fragen, welche sozialen Mechanismen den Berufen ihre ungleichheitsrelevante Wirkung verleihen. Der erste Themenblock des Sonderhefts verschafft den Leserinnen und Lesern einen Zugang zu theoretischen Grundlagen und Messkonzepten im Forschungsfeld „Berufe und soziale Ungleichheit“. In den anschließenden Themenblöcken wird anhand konkreter Forschungsfragen aufgezeigt, welches Potenzial in der hier vorgestellten Perspektive auf Mechanismen im Themenfeld Berufe und soziale Ungleichheit steckt. Die Themenblöcke befassen sich mit Fragen von Berufswahl und beruflichen Karrieren, mit dem Zusammenhang von Berufen und Geschlechterungleichheit, mit der Bedeutung von Berufen im Kontext der Debatten um Migration und Zuwanderung sowie mit dem Thema Berufe im Zeitalter der Digitalisierung und Globalisierung. Dabei handelt es sich explizit um theoretisch fundierte, empirische Beiträge, die gleichzei-

\section{Ebner $(\bowtie)$}

Institut für Soziologie, TU Braunschweig

Bienroder Weg 97, 38106 Braunschweig, Deutschland

E-Mail: c.ebner@tu-braunschweig.de

\author{
A. Haupt \\ Institut für Soziologie, Karlsruher Institut für Technologie (KIT) \\ 76128 Karlsruhe, Deutschland \\ E-Mail: andreas.haupt@kit.edu \\ B. Matthes \\ Institut für Arbeitsmarkt- und Berufsforschung (IAB) \\ Regensburger Straße 104, 90478 Nürnberg, Deutschland \\ E-Mail: Britta.Matthes@iab.de
}


tig die Vielfalt der für diese Art von Analysen verwendbaren Datengrundlagen und methodischen Zugänge aufzeigt.

Schlüsselwörter Beruf · Berufsforschung · Mechanismen · Berufswahl · Berufliche Mobilität

\title{
Occupations and Social Inequality: Introduction and Contents of the Special Issue
}

\begin{abstract}
There is a long tradition of studying the significance of occupations for social inequality. In this introductory article, we outline the contributions to this special issue of Kölner Zeitschrift für Soziologie und Sozialpsychologie in the context of the research into occupations carried out so far. The idea of the special issue is not to view occupation as a "black box", as done elsewhere, but to specifically ask what social mechanisms give occupations their inequality-generating effects. The first thematic block of the special issue provides the reader with an overview of theoretical perspectives and measures used in the research field of occupations and social inequality. The subsequent thematic blocks address specific research questions in order to illustrate the potential of emphasizing social mechanisms in the research area of occupations and social inequality. The thematic blocks cover the issues of occupational choice and careers, the connections between occupations and gender inequality, the significance of occupations in the context of the debates on migration and immigration, as well as the theme of occupations in the age of digitisation and globalisation. Our special issue brings together theoretically sound, empirical contributions that simultaneously demonstrate the diversity of the data bases and the methodological approaches that can be used for these types of analyses.
\end{abstract}

Keywords Occupation · Occupational research $\cdot$ Mechanisms · Occupational choice · Occupational mobility

\section{Ausgangslage und Ziel des Sonderhefts „Berufe und soziale Ungleichheit"،}

Nach wie vor ist der Beruf einer der wichtigsten Bestimmungsfaktoren, um Personen im sozialen Ungleichheitsgefüge der Gesellschaft zu verorten. So belegen empirische Studien beispielsweise, dass Berufe ihren Inhabern unterschiedliche Beschäftigungs- und Einkommenschancen bieten (z. B. Abraham et al. 2018; Blien und Phan Thi Hong 2010; Blossfeld 1990; Solga und Konietzka 2000; Stüber 2016), die Berufswahl entscheidend für den weiteren Erwerbsverlauf ist (z.B. Fitzenberger und Kunze 2005; Konietzka 1999; Schmillen und Möller 2012) und Berufswechsel mit einem hohen Risiko unterwertiger Beschäftigung (z. B. Mayer et al. 2010; Menze 2017) und Lohnverlusten verbunden sein können (z. B. Kracke et al. 2018; Nawakitphaitoon und Ormiston 2015).

Während der Zusammenhang zwischen Berufen und unterschiedlichsten Dimensionen sozialer Ungleichheit - wie Löhnen, Prestige, Jobstabilität, Arbeitsbedingun- 
gen, Belastungen oder Gesundheit - kaum mehr bestritten wird, gibt es in Bezug auf die Mechanismen, die hinter der ungleichheitsstiftenden Wirkung von Berufen stehen, noch erheblichen Forschungsbedarf. Worauf lässt sich beispielsweise zurückführen, dass viele Berufe, die als ,systemrelevant“ betrachtet werden - auf die also in Krisenzeiten wie der Coronapandemie 2020 nicht verzichtet werden konnte typischerweise nur gering angesehen und gering entlohnt sind (Koebe et al. 2020)? Wie lassen sich berufsspezifische Ungleichheiten in all ihren Facetten erklären?

Bereits bei den Klassikern der Soziologie (wie z. B. Durkheim 1988; Marx 1867; Parsons 1958; Weber 1972) finden sich theoretische Überlegungen zur gesellschaftlichen Bedeutsamkeit von Berufen (siehe auch Demszky und Voß 2018). Dabei wurde vor allem die makrosoziologische Bedeutung des Berufs in Bezug auf Ökonomie, Integration oder als Organisationsprinzip der gesellschaftlichen Arbeitsteilung diskutiert.

Statistisch erfassten Berufszählungen schon im 19. Jahrhundert, welche Personen welche Berufe ausübten (vgl. z. B. Stockmann und Willms-Herget 1985). Aber erst in den Statuserwerbsmodellen der 1960er- und 1970er-Jahre wurde dem Zusammenhang von Beruf und sozialer Ungleichheit größere Aufmerksamkeit geschenkt. Seitdem wird der Beruf in empirischen Analysen häufig für die Einordnung und Bewertung beruflicher Mobilitätsbewegungen verwendet (z. B. Blau und Duncan 1978) oder dazu genutzt, die Zugehörigkeit von Personen zu einer Großklasse zu bestimmen (z.B. Erikson und Goldthorpe 1992). Bei diesen Analysen werden Berufe als Indikatoren für die Messung sozialer Ungleichheit verwendet, ohne aber die diesem Zusammenhang zugrunde liegenden Mechanismen in den Blick zu nehmen.

Eine andere Perspektive auf den Zusammenhang von Beruf und sozialer Ungleichheit nahm die deutschsprachige Berufssoziologie in den 1980er-Jahren ein, indem sie bei der Betrachtung der Bedeutung des Berufs nicht mehr nur auf ,Struktur“, sondern auch auf das „Subjekt" setzte. Dies zeigt sich insbesondere in der damaligen Definition von Berufen als ,komplexe, institutionalisierte Bündelungen der marktrelevanten Arbeitsfähigkeiten von Personen“ (Beck et al. 1980, S. 19). Berufe werden damit als prägend für die soziale Statuszuweisung und die individuelle Identitätsbildung, aber auch als Personeneigenschaft entworfen. Auch hier ging es im Kern jedoch nicht darum zu fragen, worauf die Ungleichheitsrelevanz von Berufen zurückzuführen ist, sondern um die Feststellung, dass sich Berufe als Bündel an typischen Fähigkeiten, Fertigkeiten und Kenntnissen konzipieren lassen.

Der Fokus des Sonderhefts liegt im Gegensatz dazu explizit darauf aufzuzeigen, wie der Zusammenhang oder das Wechselspiel von Berufen und sozialer Ungleichheit aufgeklärt werden kann. Insgesamt ergeben sich vielfältige Möglichkeiten, die „Blackbox“ Berufe zu öffnen und die ungleichheitsrelevanten Wirkmechanismen aufzudecken. Dabei geht es nicht um ein gänzlich neues theoretisches Konzept, sondern vielmehr um mehrschichtige konzeptionelle Erweiterungen der Betrachtung von Berufen. Zum Beispiel zeigt sich, dass sich Berufe darin unterscheiden können, wie stark spezialisiert, standardisiert und institutionell fixiert die jeweiligen beruflichen Arbeitskraft- oder Tätigkeitsmuster sind (z. B. Damelang et al. 2015). Oder es gilt, das Wechselspiel der beruflichen Eigenschaften sowohl auf Individualebene als auch auf Arbeitsplatzebene in den Blick zu nehmen (z.B. Rohrbach-Schmidt und Tiemann 2016). Durch die Verknüpfung bewährter Methoden mit neuen Daten 
wird es möglich, empirische Antworten auf alte und neue Fragen der Soziologie zu geben. So werden Panelmodelle mit fixen Berufseffekten (Hausmann et al. 2015) oder Matchingverfahren (Meyer und Sacchi in diesem Sonderheft) verwendet, um die Mechanismen hinter der Veränderung geschlechtsspezifischer Entlohnung zu ergründen. Vignettenstudien erlauben es, der Berufsspezifik von Diskriminierung bei der Stellenbesetzung empirisch unmittelbar auf die Spur zu kommen (z.B. Bertogg et al. in diesem Sonderheft; Damelang und Abraham 2016; Protsch und Solga 2015) oder zu zeigen, welche Bedeutung berufsspezifische Abschlüsse in den institutionellen Kontexten verschiedener Länder haben (z.B. Di Stasio und Van De Werfhorst 2016; Stumpf et al. in diesem Sonderheft). Neue Indikatoren zur Messung berufsspezifischer Eigenschaften können verwendet werden, um viele bislang ungeprüfte Annahmen klassischer Theorien aber auch neuerer theoretischer Ansätze auf ihre Gültigkeit empirisch direkt zu testen, zum Beispiel, um die Rolle des Berufs für die berufliche Mobilität (z.B. Vicari und Unger in diesem Sonderheft), die im Zusammenhang mit Berufen stehenden sozialen Schließungsprozesse (z. B. Giesecke et al. in diesem Sonderheft; Haupt 2012) oder die Folgen von Digitalisierung (z. B. Dengler und Matthes 2018) oder Dekarbonisierung (z. B. Janser 2018) für die berufsspezifische Beschäftigungs- und Lohnentwicklung zu untersuchen.

Angesichts dieser Weiterentwicklungen ist es - nach der intensiven Diskussion um Berufe in den 1970er- und 80er-Jahren (vgl. z. B. Beck et al. 1980; Blossfeld und Mayer 1988; Lutz und Sengenberger 1974; Mertens 1973) und der erneuten Debatte über die „Erosion des Berufs“ in den 1990er-Jahren (z. B. Baethge und BaethgeKinsky 1998; Dostal et al. 1998) - an der Zeit, ein neues Sammelwerk für die (vor allem deutschsprachige) Soziologie zu schaffen, in welchem die Relevanz und das Potenzial berufsspezifischer Analysen für klassische und neue Fragen in Bezug auf soziale Ungleichheit in ihrer großen Breite verdeutlicht wird.

\section{Aufbau und Inhalte des Sonderhefts}

Die Beiträge des vorliegenden Sonderhefts vermögen zwei Dinge zu leisten. Zum einen decken sie Zusammenhänge zwischen Berufen und unterschiedlichsten Dimensionen sozialer Ungleichheit auf. Zum anderen tragen sie aber vor allem dazu bei, Mechanismen zu identifizieren, die Berufen ihre ungleichheitsstiftende Wirkung verleihen. Ungleichheitsdimensionen und Mechanismen sind dabei offenkundig je nach Untersuchungsgegenstand verschieden. Tatsächlich steht eine große Zahl gegenwärtiger gesellschaftlicher Herausforderungen direkt oder indirekt in einem Zusammenhang mit dem Thema „Beruf“. Dies gilt umso mehr für Deutschland, sind hierzulande doch nicht nur der Arbeitsmarkt, sondern auch das Bildungssystem in hohem Maße beruflich strukturiert. Dass der erlernte oder ausgeübte Beruf maßgeblich die Karriereentwicklung von Individuen prägt, liegt auf der Hand. Ein anderer Diskussionsstrang dreht sich um Fachkräftemangel, der in verschiedenen Berufen beklagt oder für die Zukunft prognostiziert wird. Um das Problem solcher Engpässe zu verstehen und zu lösen, erfordert es sowohl eine genaue Betrachtung der beruflichen Orientierungen junger Menschen (Angebotsseite) als auch der Auswahlprozesse von Arbeitgebern (Nachfrageseite). Eine weiterhin ungelöste und viel 
diskutierte gesellschaftliche Herausforderung ist die fortwährende Geschlechterungleichheit. Auch hier sind Berufe ein entscheidender Einflussfaktor. Kaum mehr aus der öffentlichen Diskussion wegzudenken ist zudem das Megathema Migration, das durch die starke Zuwanderung Geflüchteter nach Deutschland hierzulande enorm beflügelt wurde. Ausbildung und Beruf spielen in diesem Feld eine zentrale Rolle. Schließlich wird gegenwärtig auch verstärkt diskutiert, wie die Globalisierung und die Digitalisierung der Arbeitswelt Berufsstrukturen und damit Lohngefüge, aber auch die Art zu arbeiten verändert (Stichwort „Homeoffice“). All dies hat mit Berufen zu tun.

Die Beiträge des Sonderhefts greifen solche Debatten auf und bringen sie ein großes Stück voran. Insgesamt umfasst das Sonderheft 19 wissenschaftliche Beiträge. Die Datengrundlagen und methodischen Zugänge der Beiträge sind vielfältig. Jeder Beitrag wurde einem der fünf folgenden thematischen Blöcke zugeordnet:

- Block 1: Berufe - Theoretische Perspektiven und Messkonzepte

- Block 2: Berufswahl und berufliche Karrieren

- Block 3: Berufe und Geschlechterungleichheit

- Block 4: Berufe und Migration

- Block 5: Berufe, Digitalisierung und Globalisierung

Der erste Themenblock des Sonderhefts verschafft den Leserinnen und Lesern einen Einblick in Theorien zum Gegenstand „Berufe und soziale Ungleichheit“ sowie zu berufsspezifischen Messkonzepten. In den anschließenden Themenblöcken wird anhand konkreter Forschungsfragen aufgezeigt, welche Potenziale in der hier vorgestellten neuen Perspektive auf das Themenfeld Berufe und soziale Ungleichheit stecken. Die Themenblöcke befassen sich mit Fragen von Berufswahl und beruflichen Karrieren, mit dem Zusammenhang von Berufen und Geschlechterungleichheit, mit der Bedeutung von Berufen im Kontext der Debatten um Migration und Zuwanderung sowie mit dem Thema Berufe im Zeitalter der Digitalisierung und Globalisierung.

\subsection{Themenblock 1: Berufe - Theoretische Perspektiven und Messkonzepte}

Im Anschluss an diese Einleitung findet sich Themenblock 1: „Berufe - Theoretische Perspektiven und Messkonzepte“. Hier steht insbesondere im Vordergrund, einen möglichst systematischen und umfassenden Überblick über die theoretischen Grundlagen und Messkonzepte für die in diesem Sonderheft präsentierte empirische Forschung rund um das Themenfeld „Berufe und soziale Ungleichheit“ zu geben. Die beiden Beiträge bieten einerseits die Möglichkeit, sich schnell einen Überblick zu den typischen Perspektiven, Argumenten und Praktiken bei der Analyse der Bedeutung von Berufen für verschiedene soziale Ungleichheitsdimensionen $\mathrm{zu}$ verschaffen. Andererseits wird dort aber auch diskutiert, welche Potenziale verschiedene theoretische Forschungsansätze haben, um Hypothesen über die sozialen Mechanismen formulieren zu können, die die Ungleichheitsrelevanz von Berufen begründen können, oder welche berufsbasierten Skalen sich am besten für die Beantwortung spezifischer Forschungsfragen eignen. 
Der erste Beitrag, „Occupations and Inequality. Theoretical Perspectives and Mechanisms" von Andreas Haupt und Christian Ebner bestimmt das Verhältnis von Berufen und sozialer Ungleichheit aus theoretischer Sicht. Es werden vier unterschiedliche Perspektiven auf Berufe diskutiert, die sich für die Analyse sozialer Wirkmechanismen berufsspezifischer Ungleichheit als fruchtbar erwiesen haben. Dabei werden Berufe erstens als Fähigkeitsbündel, zweitens als Tätigkeitsbündel, drittens als Institutionen und viertens als kultureller Kontext konzeptualisiert. In Abhängigkeit der theoretischen Perspektive bieten sich mannigfaltige Ansatzpunkte, um mit Berufen im Zusammenhang stehende soziale Ungleichheiten zu erklären. Anhand eines Beispiels, nämlich anhand von Lohnungleichheiten, wird exemplarisch aufgezeigt, welche verschiedenen Erklärungsansätze sich auf der Grundlage der vier verschiedenen theoretischen Ansätze ergeben.

Der nachfolgende Beitrag „Occupation-Based Measures - An Overview and Discussion“"von Bernhard Christoph, Britta Matthes und Christian Ebner geht auf die Messkonzepte von Beruf und beruflichen Eigenschaften ein. Dabei werden nicht nur die weitverbreiteten berufsbasierten Instrumente zur Analyse sozialer Schichtung, wie z. B. Prestigeskalen, sozioökonomische Indizes oder Klassenschemata beschrieben, sondern auch Instrumente zur Erhebung beruflicher Tätigkeitsinhalte sowie Indikatoren zur Erfassung berufsspezifischer Gesundheitsrisiken, beruflicher Geschlechtersegregation oder beruflicher Schließung behandelt. Zudem wird ein Überblick darüber gegeben, wie Berufe in Umfragen erfasst und codiert werden, und welche Berufsklassifikationen dabei typischerweise zum Einsatz kommen.

\subsection{Themenblock 2: Berufswahl und berufliche Karrieren}

Der zweite Themenblock befasst sich mit dem Untersuchungsgegenstand „Berufswahl und berufliche Karrieren“. Die in diesem Abschnitt versammelten Beiträge analysieren die Bedeutung von Berufen für die Herausbildung sozialer Ungleichheiten zu unterschiedlichen Zeitpunkten im Lebensverlauf. Hier wird vor allem deutlich, dass Berufe ihre ungleichheitsschaffende Wirkung nicht erst auf dem Arbeitsmarkt entfalten und dass es vom Beruf abhängt, ob und in welche Richtung berufliche Entscheidungen im weiteren Erwerbsverlauf revidiert werden können. Die Beiträge in diesem Themenblock versammeln neue Erkenntnisse zur Entstehung sozialer Ungleichheiten aufgrund einer regional geprägten beruflichen Interessensformierung, aufgrund von Selektion in verschiedene Ausbildungsberufe, aufgrund berufsspezifischer Mobilitätsbarrieren sowie des ungleichen Ertrags verschiedener beruflicher Karrieren. Zudem werden hier erstmals - am Beispiel des Arbeitsmarkts für frühkindliche Bildung - Entwicklungen von Ungleichheiten im Eintritt und Verbleib von Personen unterschiedlicher Berufe innerhalb dieses Markts anhand von regulatorischen Veränderungen beschrieben.

Matthias Flohr, Laura Menze und Paula Protsch analysieren in ihrem Artikel „Berufliche Aspirationen im Kontext regionaler Berufsstrukturen“, welchen Einfluss regionale Kontexte auf die beruflichen Pläne Jugendlicher in Deutschland haben. Als Datenbasis dient das Nationale Bildungspanel (Startkohorte 4), das um beruflichregionale Indikatoren der Bundesagentur für Arbeit ergänzt wird. Mit konditionalen logistischen Regressionsmodellen kann gezeigt werden, dass Berufe stärker aspiriert 
werden, wenn diese einen höheren relativen Anteil in der Region ausmachen. Jugendliche nehmen die Berufszusammensetzung in ihrer Umgebung demnach über signifikante Andere wahr und formen entsprechende Berufswünsche. Berufswünsche sind über regionale Kontexte auch unabhängig von individuellen Eigenschaften vermittelt, was in der Literatur bis heute in dieser Form nicht klar beschrieben wurde.

Thomas Meyer und Stefan Sacchi analysieren in ihrem Beitrag ,Wieviel Schule braucht die Berufsbildung? Eintrittsdeterminanten und Wirkungen von Berufslehren mit geringem schulischen Anteil“ den Übergang von der Schule in Ausbildung und Erwerbsleben auf Basis des TREE-Datensatzes in der Schweiz. Das schweizer Schulsystem ist hoch stratifiziert. Schülerinnen und Schüler werden je nach Kanton in zwei bis vier Sekundarschul-I-Typen eingeteilt, die ein unterschiedliches Leistungsniveau bedienen (sollen). Das Schweizer Berufsausbildungssystem bietet darüber hinaus Berufslehren auf unterschiedlich hohem Niveau oder mit unterschiedlich hohem (berufs-)schulischen Anteil. Die Autoren verdeutlichen, dass Schülerinnen und Schüler, die vergleichbar leistungsfähig sind, in Abhängigkeit des besuchten Sekundarschul-I-Typs und der Bildungsarchitektur des Kantons unterschiedlich anspruchsvolle Ausbildungen aufnehmen. Der Schultyp erscheint als ein negatives Signal für Ausbildungsfähigkeit, denen entsprechende ,gate keeper“ starkes Gewicht verleihen. Wie die Autoren mithilfe eines statistischen Matchingverfahrens zeigen, übersetzt sich die Ungleichheit der Selektion in die Berufsausbildung später in weitere Ungleichheiten im Arbeitsmarkt.

Der Beitrag „No Way Out or No Way In? The Effect of Standardization, Licensing and Skills Specificity of the Initial and Target Occupations on Status Mobility“ von Basha Vicari und Stefanie Unger analysiert berufliche Mobilität von Erwerbstätigen in Deutschland. Der deutsche Arbeitsmarkt gilt als beruflich stark reguliert, was einerseits zu einer höheren Passung von Berufsanforderungen und beruflichen Kompetenzen führen kann. Andererseits können Regularien aber auch ein Mobilitätshemmnis darstellen. Vor dem Hintergrund, dass berufliche Mobilität eines der wichtigsten Vehikel für Statusmobilität ist, untersuchen die Autorinnen gezielt den Einfluss von den beruflichen Eigenschaften Standardisierung, Lizenzierung und Spezifität von Berufen. Auf Basis von Daten des Nationalen Bildungspanels (Erwachsenenkohorte) und ergänzten Informationen auf Berufsebene wird mithilfe multinomialer logistischer Regressionen gezeigt, dass Statusaufstiege vor allem aus standardisierten in nicht standardisierte, von spezifischen in unspezifische und in bestimmten Fällen von nicht lizenzierten in lizenzierte Berufe möglich sind. Mobilitätsbarrieren zeigen sich sowohl mit Blick auf Regulierungen des Ausbildungsberufs wie auch des Erwerbsberufs. In der Gesamtbetrachtung wird deutlich, dass Barrieren zwischen Berufsarbeitsmärkten nach wie vor eher selten übersprungen werden können.

Johannes Giesecke, Martin Gro $\beta$ und Stefan Stuth diskutieren in ihrem Beitrag „Occupational Closure and Wage Inequality: How Occupational Closure Effects Vary Between Workers“ kritisch die Annahme, dass alle Ausübenden eines Berufs in gleicher Weise von beruflichen Schließungsprozessen betroffen sind. Sie argumentieren, dass verschiedene Formen beruflicher Schließung (Kredentialismus, Standardisierung, Lizenzierung, Vertretung durch Berufsverbände und Gewerkschaft) 
unterschiedlich starke Auswirkung auf die Entlohnung haben. Auf Basis einer Analyse von Daten der deutschen Verdienststrukturerhebung von 2006 mithilfe eines mehrstufigen Regressionsmodells wird erkennbar, dass sich die Auswirkungen der verschiedenen, beruflichen Schließungsmechanismen für Beschäftigte in verschiedenen Konstellationen unterscheiden. So zahlt sich eine Vertretung durch Berufsverbände am meisten aus, wenn Beschäftigte Karriere machen oder durch bestimmte berufliche Schließungsprozesse Beschäftigte mit einem Hochschulabschluss stärker begünstigt werden als Beschäftigte mit einer beruflichen Qualifikation. Allerdings sind die Wechselwirkungen zwischen den beiden verschiedenen beruflichen Schließungsquellen Kredentialismus und Standardisierung äußerst komplex, sodass deren Wirkung im Rahmen des Beitrags nicht zweifelsfrei geklärt werden konnte.

Der Beitrag von Mariana Grgic „Kollektive Professionalisierungsprozesse in der Frühen Bildung - Entwicklung des Mandats, der Lizenzierung und der beruflichen Mobilität im Zeitraum 1975 bis 2018 in Westdeutschland“ nimmt langfristige Entwicklungen des ungleichen Zugangs und Verbleibs in einem spezifischen Teilarbeitsmarkt, nämlich dem für frühkindliche Bildung, in den Blick. Dieser Teilarbeitsmarkt war im analysierten Zeitraum erheblichen institutionellen Reformen unterlegen, die sowohl die Arbeitsinhalte als auch den Zugang zum Arbeitsmarkt mit unterschiedlichen Berufsabschlüssen betrafen. Auf Grundlage einer qualitativen Gesetzesanalyse zeigt die Autorin, dass der Gesetzgeber die Aufgaben für Kindergärten und Krippen zunächst als Pflegeauftrag formulierte. Dies wandelte sich jedoch immer stärker zu einem Bildungs-, Erziehungs- und Betreuungsauftrag. In vergangenen Jahren wurde schließlich der Bildungsauftrag immer stärker betont. Die Änderungen im Auftrag bedingten einen Wandel vom Jedermannsarbeitsmarkt zum berufsfachlichen Arbeitsmarkt. Die unterschiedliche Priorisierung zwischen Bildungs-, Erziehungsund Betreuungsauftrag im Zeitverlauf und zwischen den Ländern führte außerdem zu einer Heterogenität im Zugang zum Arbeitsmarkt mit unterschiedlichen Berufsabschlüssen. Im Zeitverlauf wurde der Zugang zum Teilarbeitsmarkt frühkindliche Bildung u. a. für Grundschullehrerinnen und -lehrer, für Logopäden und Logopädinnen, für Sonderpädagoginnen und -pädagogen sowie Psychologinnen und Psychologen geöffnet. Dieser multiprofessionelle Zugang geht mit einer heterogenen Lohnstreuung im Teilarbeitsmarkt einher. Der Beitrag zeigt außerdem, dass Berufsausübende bis Anfang der 1990er-Jahre sowohl ohne Abschluss als auch mit universitärem Abschluss die geringste Bleibedauer im Arbeitsmarkt haben. Die einsetzende Akademisierung des Arbeitsmarkts führte jedoch zu einer Egalisierung der Unterschiede im Verbleib zwischen akademischen und berufsfachlich ausgebildeten Berufsausübenden.

Insgesamt verdeutlichen die Beiträge des zweiten Themenblocks eindrucksvoll, wie stark die individuellen Berufschancen von beruflichen Merkmalen abhängen, die von einzelnen Akteuren kaum beeinflusst werden können. Die regionale Berufskomposition, die negative Signalwirkung von Schultypen für Ausbildungsberufe oder die Art der Regulierung von Berufen sind gesellschaftliche Phänomene, die erhebliche Auswirkungen auf die soziale Ungleichheit haben. 


\subsection{Themenblock 3: Berufe und Geschlechterungleichheit}

Berufe besitzen eine starke Verbindung zu geschlechtsspezifischer Ungleichheit. Frauen und Männer wählen systematisch andere Berufe oder werden für bestimmte Berufe mehr oder weniger in Betracht gezogen. Geschlechtsspezifische Lohnungleichheit lässt sich zu hohen Teilen auf die Geschlechtersegregation zwischen Berufen zurückführen. Einige Berufe bieten eine gute Vereinbarkeit von Arbeit und Familie, während andere Berufe eine stark ausgeprägte Vollzeit- und Überstundenkultur aufweisen, die es besonders Müttern schwermacht, in diesen Berufen Karriere zu machen. Die Beiträge des dritten Themenblocks „Berufe und Geschlechterungleichheit“ bieten neue Erkenntnisse innerhalb dieser großen Debatten. Sie stellen bestehende Annahmen, wie die Stabilität von geschlechtsspezifischen Berufswünschen, in Frage. Und sie vertiefen unser Verständnis der Prozesse, die zur starken Verbindung von Berufen mit geschlechtsspezifischer Ungleichheit führen. Jeder Beitrag lässt sich, wie im vorherigen Block, als Analyse an einem biografischen Übergang verstehen.

Der Beitrag „Zurück zur Norm? Kompromissbildung zwischen geschlechtstypischen und -untypischen Berufsaspirationen, Bewerbungs- und Ausbildungsberufen“" von Corinna Kleinert und Brigitte Schels widmet sich den Berufswünschen von Schülerinnen und Schülern in Haupt- und Realschulen. Die Autorinnen diskutieren, dass Berufswünsche nicht statisch sind, sondern sich im Bewerbungsprozess verändern. Auf Basis einer lokalen Längsschnittbefragung, die Informationen über realistische Berufsaspirationen, Bewerbungsberufe und den Beruf der ersten Ausbildung enthält, wird mithilfe von Mehrebenenmodellen gezeigt, dass Schülerinnen und Schüler am Anfang der Bewerbungsphase mit einer höheren Wahrscheinlichkeit geschlechtsuntypische Berufe aspirieren als am Ende der Bewerbungsphase. Die Autorinnen interpretieren dies als Prozess der Kompromissbildung hin zur geschlechterkonformen Norm. Sie finden bei Frauen eine stärkere Abweichung zwischen Wünschen, Bewerbungen und dem erreichten Ausbildungsberuf als bei Männern. Die Arbeit zeigt eindrücklich, dass die Wahl von Berufen keine rein individuelle Entscheidung ist, die durch geschlechtsspezifische Präferenzen bestimmt wird. Die Rückmeldungen aus der Umwelt der Schülerinnen und Schüler sowie ein höherer Erfolg in geschlechtskonformen Ausbildungsberufen tragen erheblich zur Persistenz beruflicher Geschlechtersegregation bei.

Der Beitrag „Gender Discrimination in the Hiring of Skilled Professionals in Two Male-Dominated Occupational Fields: A Factorial Survey Experiment with Real-World Vacancies and Recruiters in Four European Countries"von Ariane Bertogg, Christian Imdorf, Christer Hyggen, Dimitris Parsanoglou und Rumiana Stoilova nimmt Diskriminierungsprozesse international vergleichend für die beiden männlich dominierten Berufe Mechaniker/in und Informatiker/in unter die Lupe. Die Analysen basieren auf einer Vignettenstudie, bei der reale Stellenanzeigen gesampelt und die zuständigen Personalverantwortlichen befragt wurden. Es zeigt sich, dass Geschlechterdiskriminierung im Beruf Mechaniker/in tendenziell stärker verbreitet ist als im Beruf Informatiker/in. Als Mechanismus benennen die Autorinnen und Autoren vor allem statistische Diskriminierung. Ein besonderer Verdienst des Beitrags ist, dass er berufsspezifische Diskriminierungsprozesse in Verbindung mit 
organisationalen Charakteristika herausarbeitet. So unterscheiden sich Ergebnisse zur geschlechtsspezifischen Rekrutierung nicht nur nach Untersuchungsland, sondern auch in Abhängigkeit der nötigen Arbeitserfahrung für die zu besetzende Stelle, dem Status der Stelle (Entlohnung, Position und Arbeitszeit) sowie der wirtschaftlichen Entwicklung des Betriebs und ob es sich um eine gemeinnützige Organisation handelt oder nicht.

Geschlechtsspezifische Entlohnung ist ein weltweit verbreitetes Phänomen, das trotz intensiver Debatte um dessen Ursachen immer noch erhöhten Forschungsbedarf bietet. In ihrem Beitrag „Skill Specificity of Upper-Secondary Training Occupations and the Gender Pay Gap“ untersuchen Miriam Grønning, Irene Kriesi und Stefan Sacchi die Entlohnung von Männern und Frauen in Abhängigkeit der Fähigkeiten, die in der beruflichen Ausbildung erworben wurden. Die Auswertungen beruhen auf der schweizerischen Arbeitskräfteerhebung, die mit zusätzlichen Daten angereichert wird. Die Ergebnisse der Mehrebenenanalysen weisen auf ein komplexes Zusammenspiel zwischen Geschlecht und Fähigkeiten hin. So erhöhen berufsspezifische Kenntnisse insbesondere den Lohn bei Männern, während Frauen vor allem von einem hohen Anteil an allgemeinbildendem Unterricht in der Ausbildung profitieren. Darüber hinaus bestätigt sich die These, dass eine kulturelle Abwertung weiblicher Fähigkeiten stattfindet.

Agnieszka Althaber und Kathrin Leuze untersuchen in ihrem Beitrag „Der Einfluss der beruflichen Geschlechtersegregation und beruflicher Arbeitszeitarrangements auf Teilzeitarbeit" spezifisch den Einfluss berufsstruktureller Merkmale auf den individuellen Übergang von Vollzeit in Teilzeit. Als Datenbasis dient das Nationale Bildungspanel (Startkohorte 6) kombiniert mit aggregierten beruflichen Merkmalen auf Basis des Mikrozensus. Mithilfe von Cox Proportional Hazard-Modellen zeigen die Autorinnen, dass weniger die berufliche Geschlechtersegregation, sondern vor allem berufliche Arbeitszeitarrangements Übergänge in Teilzeit beeinflussen: Während häufige Vollzeitarbeit und Überstunden in Berufen als „Vielarbeitsnorm“ für Männer den Übergang in Teilzeit eher verhindern, gehen Frauen unter diesen Rahmenbedingungen häufiger in Teilzeit über.

In der Zusammenschau verdeutlichen die Beiträge eindrucksvoll, welcher zentrale Stellenwert Berufen bei der Erklärung geschlechtsspezifischer Ungleichheiten zukommt. Geschlechtstypische Berufswünsche bilden sich dynamisch schon früh im Lebenslauf heraus, wobei junge Menschen ihre beruflichen Aspirationen in enger Abstimmung mit der (beruflichen) Umwelt entwickeln. Prozesse statistischer Diskriminierung von Männern und Frauen spielen beim Zugang zu Berufen eine Rolle; Unterschiede sind nach Beruf, organisationalen Rahmenbedingungen und dem Länderkontext erkennbar. Um Karrieren und Löhne von Männern und Frauen besser $\mathrm{zu}$ verstehen, weisen die Beiträge darüber hinaus sowohl auf berufsspezifisch erlernte Fähigkeiten und Fertigkeiten wie auch auf berufsspezifische Normen (z.B. Überstunden machen) hin.

\subsection{Themenblock 4: Berufe und Migration}

Der vierte Themenblock befasst sich mit dem Themenfeld „Berufe und Migration“. Die Integration von Migrantinnen und Migranten in den Arbeitsmarkt hält für die 
Länder Europas Chancen und Herausforderungen bereit. In der deutschen Geschichte haben Migrantinnen und Migranten nach dem Zweiten Weltkrieg in erheblichem Maße dazu beigetragen, Fachkräfteengpässe auszugleichen und damit nicht nur zur Stabilisierung, sondern auch zum Erfolg der deutschen Wirtschaft beigetragen. Eine Herausforderung stellen heute vor allem die teils sehr unterschiedlichen Ausbildungsmodalitäten der Herkunftsländer und damit die berufsspezifischen Zertifikate und „,skillsets“ von Personen mit Migrationshintergrund dar. Wie Verantwortliche im Personalwesen damit umgehen und welche Chancen Migrantinnen und Migranten unter verschiedenen Arbeitsmarktbedingungen haben, stellen die nachfolgenden Beiträge eindrucksvoll dar.

Der Beitrag „How National Institutions Shape Skilled Immigrants' Chances of Getting Hired: Evidence from Harmonized Factorial Surveys with Employers in Germany and England“ von Felix Stumpf, Andreas Damelang, Martin Abraham und Sabine Ebensperger betont länderspezifische Rahmenbedingungen für den Berufserfolg von Migrantinnen und Migranten. Mithilfe einer experimentellen Studie für Bürokaufleute in Deutschland und England wird untersucht, ob die institutionelle Ausgestaltung der Ausbildungssysteme im Herkunfts- und Aufnahmeland die Rekrutierungschancen beeinflusst. Ausbildungssysteme des Herkunftslands bestimmen den Typus beruflicher Qualifikationen, die ausländische Fachkräfte mitbringen, und prägen im Aufnahmeland, welche Qualifikationen Arbeitgeber bei der Stellenbesetzung erwarten. Es zeigt sich, dass migrantische Bewerberinnen und Bewerber eher zum Vorstellungsgespräch für qualifizierte Jobs eingeladen werden, wenn sie aus Ländern mit hochgradig standardisierten und berufsspezifischen Ausbildungssystemen kommen. Dies gilt auch unabhängig vom Ausbildungssystem des Aufnahmelands.

In ihrem Beitrag „Licensing, Educational Credentialing and Wages Among Foreign Skilled Workers in Germany" untersucht Daniela Rohrbach-Schmidt die Erwerbschancen von im Ausland ausgebildeten Fachkräften in Deutschland. Eine Auswertung der BIBB/BAuA Erwerbstätigenbefragungen 2006, 2012 und 2018 unter Nutzung eines Reweightingansatzes ergibt, dass staatlich regulierte, lizenzierte sowie kredentialistisch geschlossene Berufe eine Hürde beim Berufszugang für im Ausland ausgebildete Fachkräfte darstellen. Ist diese Hürde jedoch genommen, findet die Studie im Durchschnitt zumindest für im Ausland qualifizierte Männer keine Lohnnachteile mehr, was die Autorin auf die deutliche Signalwirkung von Qualifikationen in regulierten Berufen zurückführt. In weniger geschlossenen Segmenten des Arbeitsmarkts zeigen sich dagegen erhebliche Lohnnachteile für ausländisch qualifizierte Erwerbstätige.

Marvin Bürmann untersucht in seinem Beitrag „,Schließung durch berufliche Standardisierung? Zur Signalwirkung in- und ausländischer Abschlüsse“ die formale Unterqualifikation bei Beschäftigten. Als unterqualifiziert gelten Personen, die einer Tätigkeit mit höherem Anforderungsprofil nachgehen als es ihr formaler Abschluss erwarten ließe. Berechnungen auf Basis des Sozio-oekonomischen Panels zeigen, dass eine formale Unterqualifikation bei Beschäftigten mit berufsqualifizierenden Abschlüssen umso seltener ist, je standardisierter ein Beruf ist. Nicht feststellbar ist dieser Effekt jedoch bei Beschäftigten ohne berufsqualifizierende Abschlüsse. Der Grad der Standardisierung wirkt sich bei Personen mit Migrationshintergrund zudem 
genauso stark auf Unterqualifikation aus wie bei Personen ohne Migrationshintergrund. Die Studie findet damit keine Hinweise darauf, dass Personalverantwortliche Abschlüsse von Personen mit Migrationshintergrund besonders abwerten (oder aufwerten). Allerdings gelingt Personen mit Migrationshintergrund der Zugang zu den stärker geschlossenen, aber monetär attraktiveren berufsspezifischen Arbeitsmärkten seltener.

Insgesamt zeigt sich, dass die Chancen zur Arbeitsmarktintegration bei Migrantinnen und Migranten von der Ausgestaltung der beruflichen Ausbildungssysteme ihres Herkunftslands abhängen. Darüber hinaus können Berufe, die im Aufnahmeland reguliert, lizenziert oder anderweitig kredentialistisch geschlossen sind, hohe Hürden beim Berufszugang für Migrantinnen und Migranten darstellen. Sind solche Hürden genommen, sind Benachteiligungen, zumindest in Deutschland, nicht mehr klar erkennbar.

\subsection{Themenblock 5: Berufe, Digitalisierung und Globalisierung}

Der Einsatz digitaler Technologien kann dazu führen, dass bestimmte berufliche Tätigkeiten nicht mehr von Menschenhand, sondern von Computern und computergesteuerten Maschinen erledigt werden. Die Digitalisierung kann aber auch dazu beitragen, dass die Arbeitsqualität steigt, indem zum Beispiel Arbeitsbelastungen reduziert werden oder die Arbeit im Homeoffice erledigt werden kann. Neben der Digitalisierung stellt die Globalisierung einen weiteren Megatrend dar, von dem Berufsausübende mehr oder weniger stark negativ betroffen sind oder aber auch profitieren. So kann, indem beispielsweise bestimmte berufliche Tätigkeiten im Ausland erledigt werden können, die Nachfrage nach diesen Berufen im Inland sinken. Andererseits kann ein erhöhter Export von Gütern aber auch zu einer gesteigerten Nachfrage nach Berufen führen, deren Tätigkeiten mit der Herstellung dieser Exportgüter in Verbindung stehen. All das hat Auswirkungen auf die Berufsstruktur, die Beschäftigungschancen und das Lohngefüge, aber auch auf die Art zu arbeiten. In den Beiträgen im fünften Themenblock „Berufe, Digitalisierung und Globalisierung“ werden, bezugnehmend auf diese beiden Diskussionsstränge, neue Ergebnisse zum Verhältnis von Beruf und sozialer Ungleichheit präsentiert. So wird erstmals diskutiert, ob sich durch die digitale Transformation die geschlechtsspezifischen Ungleichheiten in den berufsspezifischen Arbeitsbelastungen verstärken könnten; warum die in einem Beruf neu hinzukommenden IT-Kenntnisse besser entlohnt werden als IT-Kenntnisse, die bereits zentraler Bestandteil des beruflichen Qualifikationsbündels sind; warum gerade Beschäftigte, die in Routinejobs arbeiten, seltener an Weiterbildung teilnehmen; welche berufsspezifischen Ungleichheiten es beim Zugang zu Homeoffice gibt; und warum internationale Handelsverflechtungen Auswirkungen auf das berufliche Lohngefüge haben.

Im Beitrag „Examining the Relationship Between Digital Transformation and Work Quality: Substitution Potential and Work Exposure in Gender-Specific Occupations“" analysieren Katharina Dengler und Anita Tisch den Zusammenhang zwischen Digitalisierung und Arbeitsbelastungen in männlich und weiblich dominierten Berufen. Sie kombinieren Informationen aus verschiedenen Datensätzen auf der beruflichen Ebene: administrative Daten der IAB-Beschäftigungshistorien $(\mathrm{BeH})$, 
Berufsinformationen aus dem BERUFENET sowie Daten aus der BIBB/BAuA-Erwerbstätigenbefragung und untersuchen mithilfe einer OLS-Regressionsanalyse, ob sich im Zuge der digitalen Transformation die bereits vorhandenen Ungleichheiten bezüglich der Arbeitsbelastungen zwischen Frauen und Männern weiter verschärft haben. Es zeigt sich, dass durch den Einsatz digitaler Technologien Berufe mit hohen psychosozialen Arbeitsbelastungen nicht entlastet werden können, während in Berufen mit hohen physischen Arbeitsbelastungen der Anteil körperlich anstrengender Tätigkeiten abnehmen könnte. Potenziell könnte es also durch die Digitalisierung zu einer steigenden Ungleichheit zwischen Frauen und Männern bezogen auf die Arbeitsbelastungen kommen. Allerdings hat sich die Digitalisierung bislang (noch) nicht in einem Rückgang der Beschäftigung in Berufen mit hoher physischer Arbeitsexposition niedergeschlagen, sodass man derzeit keine Anzeichen für eine zunehmende Ungleichheit erkennen kann.

In ihrem Beitrag „Occupational Inequality in Wage Returns to Employer Demand for Types of Information and Communications Technology (ICT) Skills: 1991-2017““ gehen Marlis Buchmann, Helen Buchs und Ann-Sophie Gnehm der Frage nach, ob IT-Kenntnisse, die bereits ein zentraler Bestandteil des beruflichen Qualifikationsbündels sind, anders entlohnt werden als IT-Kenntnisse, die erst neu hinzugekommen sind. Mit Daten des Schweizer Stellenmarktmonitor (SJMM), die mit Lohndaten der Schweizerischen Arbeitskräfteerhebung (SAKE) verknüpft wurden, zeigen sie mithilfe von Mehrebenenmodellen, bei denen die Entlohnung und andere Kontrollvariablen auf individueller Ebene und die Nachfrage nach den verschiedenen Arten von IT-Kenntnissen auf beruflicher Ebene gemessen werden, dass die in einem Beruf neu eingeführten IT-Kenntnisse mit einer höheren Entlohnung einhergehen, während dies bei IT-Kenntnissen, die integrale Bestandteile des beruflichen Qualifikationsbündels darstellen, nicht der Fall ist. Dadurch ergeben sich im Zuge der digitalen Transformation Verschiebungen hinsichtlich der berufsbedingten Ungleichheiten bezüglich der Entlohnung.

Der Frage, warum gerade Beschäftigte in Berufen, die einem höheren Automatisierungsrisiko ausgesetzt sind, seltener an der Weiterbildung teilnehmen, geht Martin Ehlert in seinem Beitrag ,No Future, No Training? Explaining Cross-national Variation in the Effect of Job Tasks On Training Participation" nach. In einer international vergleichenden Perspektive zeigt er anhand von Daten aus dem ,Programme for the International Assessment of Adult Competencies" (PIAAC) auf, dass es einen engen Zusammenhang zwischen den auf dem Arbeitsplatz zu erledigenden Aufgaben und der Beteiligung an non-formaler Weiterbildung gibt. In einer länderübergreifenden, mehrstufigen Regressionsanalyse belegt er, dass die Weiterbildungsteilnahme auch mit dem Typ des in dem jeweiligen Land existierenden Bildungssystems im Zusammenhang steht. In Ländern mit einem weniger gegliederten Schulsystem nehmen Beschäftigte, die Routineaufgaben erledigen, häufiger an Weiterbildung teil als in Ländern mit einem stärker gegliederten Schulsystem. Dagegen führt eine stärkere Beruflichkeit des Ausbildungssystems nicht zu einer größeren Ungleichheit bei der Weiterbildungsbeteiligung. Starke Gewerkschaften und dynamische Arbeitsmärkte verbessern darüber hinaus den Zugang zur Weiterbildung für die in Routinejobs Beschäftigten. 
Dem Thema, welche berufsspezifischen Ungleichheiten beim Zugang zu Homeoffice festzustellen sind, widmet sich Alexandra Mergener in ihrem Beitrag „Berufliche Zugänge zum Homeoffice“. Auf Basis der BIBB/BAuA-Erwerbstätigenbefragung aus dem Jahr 2018 untersucht sie, welche Art beruflicher Tätigkeiten typischerweise im Homeoffice erledigt werden (können). Die Ergebnisse der Random-Effects-Modelle zeigen, dass die Chance eines Homeofficezugangs mit dem Ausmaß kognitiver Tätigkeiten bei der Arbeit steigt, während sie mit steigendem Ausmaß manueller Tätigkeiten sinkt. Darüber hinaus hängt die Stärke des Einflusses der beruflichen Tätigkeiten auf den Homeofficezugang auch von der Betriebsgröße ab. In Berufe mit einer hohen Intensität kognitiver Tätigkeiten ist die Chance, einen Homeofficezugang zu haben, in Großbetrieben mit mehr als 250 Beschäftigten deutlich höher als in Kleinbetrieben. Der negative Effekt bei der Erledigung manueller Tätigkeiten ist dagegen in mittleren oder großen Betrieben stärker als in kleinen Betrieben.

In ihrem Beitrag „Trade and Wage Inequality: The Mediating Roles of Occupations in Germany“ untersuchen Malte Reichelt, Samreen Malik und Marvin Suesse, welche Mechanismen hinter dem Befund stehen, dass die zunehmende Lohnungleichheit in vielen Industrieländern partiell auf internationale Handelsverflechtungen zurückzuführen ist. Sie analysieren die Bedeutung von Handelsbeziehungen zwischen China und Deutschland in den Jahren 1994 bis 2010 für die Lohnungleichheit in Deutschland. Mithilfe von Instrumentenvariablenschätzern anhand administrativer IAB-Beschäftigten- und Betriebsdaten (BHP, IEB), die mit Daten zum internationalen Handelsverkehr (COMTRADE) angereichert wurden, zeigen sie, dass Importe die Lohnungleichheit insgesamt nicht beeinflussen. Stattdessen sind steigende Exporte nach China für die wachsende Lohnstreuung in Arbeitsmarktregionen verantwortlich.

Abschließend ist festzustellen, dass der Zusammenhang zwischen Berufen und sozialer Ungleichheit auch den Kräften von Digitalisierung und Globalisierung ausgesetzt ist. Die Technologisierung und Computerisierung beruflicher Tätigkeiten verändert Lohngefüge und Belastungen am Arbeitsplatz und hat zudem Einfluss auf die bereits weiter oben skizzierten berufsspezifischen Geschlechterungleichheiten. Je nachdem, welche Tätigkeiten für Berufe typisch sind, bieten sich Beschäftigten unterschiedliche Möglichkeiten auf Weiterbildung oder ihre Erwerbsarbeit auch an einem anderen Arbeitsplatz als im Betrieb zu verrichten (Stichwort „Homeoffice“).

\section{Fazit und Ausblick}

Seit langer Zeit verdeutlichen empirische Studien, dass Berufe hoch mit zahlreichen Dimensionen sozialer Ungleichheit korrelieren. Weniger abstrakt gesprochen heißt dies für die Mitglieder einer Gesellschaft, dass die Ausbildung in einem bestimmten Beruf oder die spezifische Berufsausübung erkennbar über individuelle Ressourcen und Status bestimmt. Beschäftigungssicherheit, Löhne, Arbeitsbedingungen, Weiterbildungsmöglichkeiten oder Gesundheit sind also maßgeblich auch eine Frage des Berufs. 
Wir haben dies im Rahmen dieses Sonderhefts zum Anlass genommen, mehr über die ungleichheitsstiftende Wirkung von Berufen erfahren zu wollen. Während die Forschung zu Berufen in Deutschland und international bereits eine lange Tradition hat, gibt es hinsichtlich der Mechanismen, die hinter der ungleichheitsstiftenden Wirkung von Berufen stehen, Klärungsbedarf. Der Schwerpunkt des Sonderhefts liegt also explizit darauf, den Zusammenhang zwischen Berufen und sozialer Ungleichheit zu erklären oder aufzuklären.

Die einzelnen Beiträge des Sonderhefts zeigen eindrucksvoll, welche Potenziale in der hier vorgestellten neuen Perspektive auf das Themenfeld Berufe und soziale Ungleichheit stecken. Die „Blackbox“ Beruf wird dabei auf sehr unterschiedliche Art und Weise geöffnet. Das Sonderheft gibt einen ausführlichen, aber keineswegs vollständigen Einblick in die verschiedenen Themen und Fragen, die zur Untersuchung der ungleichheitsstiftenden Mechanismen von Berufen analysiert werden können. Die Vielfalt möglicher theoretischer und methodischer Zugänge sowie die für diese Art von Analysen verwendbaren Datengrundlagen kann auch nicht erschöpfend aufgezeigt werden. Vielmehr stehen die in diesem Sonderheft versammelten Beiträge als gute Beispiele dafür, wie der Perspektivwechsel zu einem besseren Verständnis des Zusammenhangs von Beruf und sozialer Ungleichheit, zur Bewältigung solcher gesellschaftlicher Herausforderungen wie Globalisierung, Migration, Digitalisierung und demografischem Wandel sowie der Erhöhung der Arbeitsqualität oder der Herstellung von Geschlechtergerechtigkeit beitragen kann.

Danksagung Wir danken allen Autorinnen und Autoren für ihre wertvollen Beiträge zum vorliegenden Sonderheft. Darüber hinaus gilt unser Dank der Redaktion der Kölner Zeitschrift für Soziologie und Sozialpsychologie - namentlich Volker Dreier - für die professionelle begleitende Unterstützung. Das Konzept für das Sonderheft wurde im Vorfeld mit mehreren Personen aus dem Herausgebergremium der Zeitschrift kritisch diskutiert. Explizit möchten wir uns für konstruktive Anregungen zur konzeptionellen Schärfung des Sonderhefts bei Hans-Jürgen Andreß, Daniela Grunow, Thomas Schwinn und Michael Wagner bedanken.

Funding Open Access funding provided by Projekt DEAL.

Open Access Dieser Artikel wird unter der Creative Commons Namensnennung 4.0 International Lizenz veröffentlicht, welche die Nutzung, Vervielfältigung, Bearbeitung, Verbreitung und Wiedergabe in jeglichem Medium und Format erlaubt, sofern Sie den/die ursprünglichen Autor(en) und die Quelle ordnungsgemäß nennen, einen Link zur Creative Commons Lizenz beifügen und angeben, ob Änderungen vorgenommen wurden.

Die in diesem Artikel enthaltenen Bilder und sonstiges Drittmaterial unterliegen ebenfalls der genannten Creative Commons Lizenz, sofern sich aus der Abbildungslegende nichts anderes ergibt. Sofern das betreffende Material nicht unter der genannten Creative Commons Lizenz steht und die betreffende Handlung nicht nach gesetzlichen Vorschriften erlaubt ist, ist für die oben aufgeführten Weiterverwendungen des Materials die Einwilligung des jeweiligen Rechteinhabers einzuholen.

Weitere Details zur Lizenz entnehmen Sie bitte der Lizenzinformation auf http://creativecommons.org/ licenses/by/4.0/deed.de. 


\section{Literatur}

Abraham, Martin, Andreas Damelang und Andreas Haupt. 2018. Berufe und Arbeitsmarkt. In Arbeitsmarktsoziologie: Probleme, Theorien, empirische Befunde, Hrsg. Martin Abraham und Thomas Hinz, 225-259. Wiesbaden: Springer Fachmedien Wiesbaden.

Baethge, Martin, und Volker Baethge-Kinsky. 1998. Jenseits von Beruf und Beruflichkeit? Neue Formen von Arbeitsorganisation und Beschäftigung und ihre Bedeutung für eine zentrale Kategorie gesellschaftlicher Integration. Mitteilungen aus der Arbeitsmarkt- und Berufsforschung 3:461-472.

Beck, Ulrich, Michael Brater und Hansjürgen Daheim. 1980. Soziologie der Arbeit und der Berufe. Grundlagen, Problemfelder, Forschungsergebnisse. Reinbeck bei Hamburg: Rowohlt.

Blau, Peter, und Otis Dudley Duncan. 1978. The American Occupational Structure (Reprint from 1967). New York: Free Press.

Blien, Uwe, und Van Phan Thi Hong. 2010. Berufliche Lohnunterschiede. In Berufsforschung für eine moderne Berufsbildung. Stand und Perspektiven (Beiheft 24 der Zeitschrift für Berufs- und Wirtschaftspädagogik), Hrsg. Dieter Euler, Ulrich Walwei und Reinhold Weiß, 263-280. Stuttgart: Steiner.

Blossfeld, Hans-Peter. 1990. Berufsverläufe und Arbeitsmarktprozesse. Ergebnisse sozialstruktureller Längsschnittuntersuchungen. Kölner Zeitschrift für Soziologie und Sozialpsychologie, Sonderheft 31:118-145.

Blossfeld, Hans-Peter, und Karl Ulrich Mayer. 1988. Labor market segmentation in the Federal Republic of Gemany: an empirical study of segmentation theories from a life course perspective. European Sociological Review 4:123-140.

Damelang, Andreas, und Martin Abraham. 2016. You Can Take Some of It with You! Zeitschrift für Soziologie 45:91-106.

Damelang, Andreas, Florian Schulz und Basha Vicari. 2015. Institutionelle Eigenschaften von Berufen und ihr Einfluss auf berufliche Mobilität in Deutschland. Schmollers Jahrbuch 135:307-333.

Demszky, Alma, und Günter G. Voß. 2018. Beruf und Profession. In Handbuch Arbeitssoziologie: Band 2: Akteure und Institutionen, Hrsg. Fritz G Böhle, Günter Voß und Günther Wachtler, 477-538. Wiesbaden: Springer Fachmedien Wiesbaden.

Dengler, Katharina, und Britta Matthes. 2018. The impacts of digital transformation on the labour market: Substitution potentials of occupations in Germany. Technological Forecasting and Social Change 137:304-316.

Dostal, Werner, Friedemann Stooß und Lothar Troll. 1998. Beruf. Auflösungstendenzen und erneute Konsolidierung. Mitteilungen aus der Arbeitsmarkt- und Berufsforschung 31:438-460.

Durkheim, Emile. 1988. Über soziale Arbeitsteilung. Studie über die Organisation höherer Gesellschaften (französisches Original 1893). Frankfurt/Main: Suhrkamp.

Erikson, Robert, und John H. Goldthorpe. 1992. The constant flux: A study of class mobility in industrial societies. University of California: Clarendon Press.

Fitzenberger, Bernd, und Astrid Kunze. 2005. Vocational Training And Gender: Wages And Occupational Mobility Among Young Workers. Oxford Review of Economic Policy 21:392-415.

Haupt, Andreas. 2012. (Un)Gleichheit durch soziale Schließung. Kölner Zeitschrift für Soziologie und Sozialpsychologie 64:729-753.

Hausmann, Ann-Christin, Corinna Kleinert und Kathrin Leuze. 2015. Entwertung von Frauenberufen oder Entwertung von Frauen im Beruf? Kölner Zeitschrift für Soziologie und Sozialpsychologie 67:217-242.

Janser, Markus. 2018. The greening of jobs in Germany. First evidence from a text mining based index and employment register data. (IAB-Discussion Paper 14/2018). Nürnberg: Institut für Arbeitsmarkt- und Berufsforschung.

Koebe, Josefine, Claire Samtleben, Annekatrin Schrenker und Aline Zucco. 2020. Systemrelevant und dennoch kaum anerkannt: Das Lohn- und Prestigeniveau unverzichtbarer Berufe in Zeiten von Corona. DIW aktuell 28.

Konietzka, Dirk. 1999. Die Verberuflichung von Marktchancen. Zeitschrift für Soziologie 28:379-400.

Kracke, Nancy, Malte Reichelt und Basha Vicari. 2018. Wage Losses Due to Overqualification: The Role of Formal Degrees and Occupational Skills. Social Indicators Research 139:1085-1108.

Lutz, Burkart, und Werner Sengenberger. 1974. Arbeitsmarktstrukturen und öffentliche Arbeitsmarktpolitik: eine kritische Analyse von Zielen und Instrumenten (Schriften der Kommission für wirtschaftlichen und sozialen Wandel 26). Göttingen: Schwartz.

Marx, Karl. 1867. Das Kapital. Band 1. Berlin: Dietz-Verlag. 
Mayer, Karl Ulrich, Daniela Grunow und Natalie Nitsche. 2010. Mythos Flexibilisierung? Wie instabil sind Berufsbiografien wirklich und als wie instabil werden sie wahrgenommen? Kölner Zeitschrift für Soziologie und Sozialpsychologie 62:369-402.

Menze, Laura. 2017. Horizontale und vertikale Adäquanz im Anschluss an die betriebliche Ausbildung in Deutschland. Kölner Zeitschrift für Soziologie und Sozialpsychologie 69:79-107.

Mertens, Dieter. 1973. Der unscharfe Arbeitsmarkt. Mitteilungen aus der Arbeitsmarkt- und Berufsforschung 6:314-325.

Nawakitphaitoon, Kritkorn, und Russell Ormiston. 2015. Occupational human capital and earnings losses of displaced workers: does the degree of similarity between pre- and post-displacement occupations matter? Journal for Labour Market Research 48:57-73.

Parsons, Talcott. 1958. Struktur und Funktion der modernen Medizin. Eine soziologische Analyse. Kölner Zeitschrift für Soziologie und Sozialpsychologie, Sonderheft 3:10-57.

Protsch, Paula, und Heike Solga. 2015. How Employers Use Signals of Cognitive and Noncognitive Skills at Labour Market Entry: Insights from Field Experiments. European Sociological Review 31:521-532.

Rohrbach-Schmidt, Daniela, und Michael Tiemann. 2016. Educational (Mis)match and skill utilization in Germany: Assessing the role of worker and job characteristics. Journal for Labour Market Research 49:99-119.

Schmillen, Achim, und Joachim Möller. 2012. Distribution and determinants of lifetime unemployment. Labour Economics 19:33-47.

Solga, Heike, und Dirk Konietzka. 2000. Das Berufsprinzip des deutschen Arbeitsmarktes: Ein geschlechtsneutraler Allokationsmechanismus? Schweizerische Zeitschrift für Soziologie 26:111-147.

Di Stasio, Valentina, und Herman G. Van De Werfhorst. 2016. Why Does Education Matter to Employers in Different Institutional Contexts? A Vignette Study in England and the Netherlands. Social Forces 95:77-106.

Stockmann, Reinhard, und Angelika Willms-Herget. 1985. Erwerbsstatistik in Deutschland: die Berufsund Arbeitsstättenzählungen seit 1875 als Datenbasis der Sozialstrukturanalyse. Frankfurt/Main, New York: Campus.

Stüber, Heiko. 2016. Berufsspezifische Lebensentgelte: Qualifikation zahlt sich aus (IAB-Kurzbericht 7/2016). Nürnberg: Institut für Arbeitsmarkt- und Berufsforschung.

Weber, Max. 1972. Wirtschaft und Gesellschaft: Grundriss der verstehenden Soziologie. Tübingen: J. C. B. Mohr.

Christian Ebner 1978, Dr. phil., Professor für Soziologie mit Schwerpunkt Arbeit und Organisation, Technische Universität Braunschweig. Forschungsgebiete: Arbeitsmarktungleichheit, berufliche Aus- und Weiterbildung, Wandel der Arbeitswelt, Berufe und Arbeitsbedingungen. Veröffentlichungen: Weiterbilden und Weiterkommen? Non-formale berufliche Weiterbildung und Arbeitsmarktmobilität in Deutschland, Kölner Zeitschrift für Soziologie und Sozialpsychologie 70, 2018 (mit M. Ehlert); Social distance and wage inequalities for immigrants in Switzerland, Work, employment and society, 30, 2016 (mit M. Helbling); Labour market developments and their significance for VET in Germany: An overview, Research in Comparative \& International Education, 10, 2015.

Andreas Haupt 1983, Dr. phil., Akademischer Rat am Karlsruher Institut für Technologie. Forschungsgebiete: Soziale Ungleichheit, Arbeitsmarkt- und Berufsforschung, Methodenforschung. Veröffentlichungen: Is occupational licensing more beneficial for women? The case of Germany, 1993 to 2015, European Sociological Review, 2019 (mit N. Witte); The Long Road to Economic Independence of German Women, 1973 to 2011, Socius 2019; Erhöhen Berufliche Lizenzen Verdienste und die Verdienstungleichheit? Zeitschrift für Soziologie 45, 2016.

Britta Matthes 1969, Dr.phil., Leiterin der Forschungsgruppe Berufliche Arbeitsmärkte am Institut für Arbeitsmarkt- und Berufsforschung (IAB). Forschungsgebiete: Berufsforschung, Genderforschung, Lebensverlaufsforschung, digitale und ökologische Transformation. Veröffentlichungen: Berufe und wie sie klassifiziert werden können. S. 79-93 in Beruf, Beruflichkeit, Employability (Hrsg. J. Seifried et al.); Do German works councils counter or foster the implementation of digital technologies?, Jahrbücher für Nationalökonomie und Statistik 239, 2019 (mit. S. Genz u. L. Bellmann); The impacts of digital transformation on the labour market. Substitution potentials of occupations in Germany, Technological Forecasting and Social Change 137, 2018 (mit K. Dengler). 